Fragment Yields Calculated in a Time-Dependent Microscopic Theory of Fission

W. Younes, D. Gogny

October 1, 2012 
This document was prepared as an account of work sponsored by an agency of the United States government. Neither the United States government nor Lawrence Livermore National Security, LLC, nor any of their employees makes any warranty, expressed or implied, or assumes any legal liability or responsibility for the accuracy, completeness, or usefulness of any information, apparatus, product, or process disclosed, or represents that its use would not infringe privately owned rights. Reference herein to any specific commercial product, process, or service by trade name, trademark, manufacturer, or otherwise does not necessarily constitute or imply its endorsement, recommendation, or favoring by the United States government or Lawrence Livermore National Security, LLC. The views and opinions of authors expressed herein do not necessarily state or reflect those of the United States government or Lawrence Livermore National Security, LLC, and shall not be used for advertising or product endorsement purposes.

This work performed under the auspices of the U.S. Department of Energy by Lawrence Livermore National Laboratory under Contract DE-AC52-07NA27344. 


\title{
Fragment yields calculated in a time-dependent microscopic theory of fission
}

\author{
W. Younes and D. Gogny
}

September 28, 2012

\begin{abstract}
Pre-neutron fragment-yield distributions are calculated for the ${ }^{235} \mathrm{U}(n, f)$ and ${ }^{239} \mathrm{Pu}(n, f)$ reactions using a time-dependent fully microscopic approach. These yields are calculated as a function of incident neutron energy from thermal to $5 \mathrm{MeV}$. We show that our calculations are in good agreement with experimental results, and competitive in accuracy with more phenomenological approaches. Further improvements to future calculations of these yields are discussed.
\end{abstract}

\section{Introduction}

Microscopic methods, where the nucleus is described starting from protons, neutrons, and an effective interaction between them, have been used to calculate a plethora of nuclear properties (see, e.g., $[1,2,3]$ and references therein). Nuclear fission in particular, has been studied using microscopic approaches since the 1980's (e.g., $[4,6,7,5,8])$. For the present work, we have adopted the Bohr approximation to the Time-Dependent Generator Coordinate Method (TDGCM), successfully used by Berger et al. [4] and Goutte et al. [6] to calculate fission as the propagation of a collective wave packet built on microscopic nuclear states and representing the fissioning nucleus as it moves toward scission. These earlier calculations were used to examine properties such as the fission time scale, and the mass distribution of fission fragments. Other, semi-classical, approaches have been used to calculate fission-fragment properties. We note in particular the full Langevin calculations of the Omsk group [9, 10, 11], and more recently the Brownian-motion approach of Randrup et al. [12,13]. We have preferred to adopt the microscopic approach for the present work because, although it is far more technically and conceptually challenging, this approach incorporates the tenets of quantum mechanics from the start, such as the non-locality of the nuclear wave function and exchange terms in the Hamiltonian of the system. These quantum-mechanical aspects of fission become particularly important near scission, as one parent nucleus divides into two fragments, each a nucleus in its own right. 
Using the TDGCM, we have calculated the fragment mass distributions, before prompt neutron emission, for the ${ }^{235} \mathrm{U}(n, f)$ and ${ }^{239} \mathrm{Pu}(n, f)$ reactions, and for incident neutron energies $E_{n}$ ranging from thermal to $5 \mathrm{MeV}$. The same D1S finite-range interaction $[14,15]$ has been used for both reactions and for all incident neutron energies. Previous work [6] has shown the importance of both the time-dependent and quantum-mechanical aspects of the theory to correctly reproduce the width of observed mass distributions through the spreading of the collective wave packet. In more phenomenological approaches (e.g., [16]) the effect of this quantum spreading is sometimes mimicked by coupling a quantum harmonic oscillator to a heat bath at finite temperature. In contrast, the spreading in = microscopic calculations is caused by the coupling between the collective degrees of freedom of the system. Thus, the choice of the collective degrees of freedom of the fissioning nucleus plays a crucial role in the calculation of the mass distributions. Until now, microscopic calculations of fission have systematically used the multipole moments of the nucleus as collective coordinates (usually the quadrupole moment $Q_{20}$ to describe its elongation, and the octupole moment $Q_{30}$ to describe the mass asymmetry between heavy and light fragments).

After an extensive analysis of TDGCM calculations of fragment distributions using the $Q_{20}$ and $Q_{30}$ coordinates, we were led to the conclusion that these coordinates, although very useful in describing the qualitative features of fission observables (see, e.g., $[6,17]$ ), are not ideal near, at, or beyond scission. We discovered that, no matter what reasonable prescription we used to relate the $Q_{20}$ and $Q_{30}$ coordinates to a fragment mass number, some fragment masses were severely under-represented in the yield distribution. In particular, for ${ }^{239} \mathrm{Pu}(n, f)$, fissions corresponding to a heavy fragment in the range $A=135-$ 142 (near the peak of the yield distribution) were very difficult to populate. As a result, the experimental mass distributions for thermal fission could only be reproduced to within a factor of two, even near the peak of those yields. It became clear that collective coordinates better suited to the description of the nucleus near scission were needed in order to reproduce the experimental mass distributions.

Instead of quadrupole and octupole moments of the nucleus, we have therefore adopted as collective coordinates the separation distance $(d)$ between the two fragments and the relative difference $(\xi)$ between their mass numbers. We have already alluded to the importance of collective coordinates describing the fragments themselves when extracting their properties, such as their excitation and kinetic energies [18], but to our knowledge this represents the first calculations of fission-fragment distributions in these new coordinates. In addition, we account for the smearing effect of particle number fluctuations on the fragment yields. In this report, we will show that these new improved calculations produce fragment yields that are generally within $\sim 30 \%$ of experimental measurements, which is comparable to more phenomenological approaches. We will also propose improvements that could further reduce the discrepancy between theory and experiment.

After a brief presentation of the formalism in section 2, the fission fragment 
distributions obtained for the ${ }^{235} \mathrm{U}(n, f)$ and ${ }^{239} \mathrm{Pu}(n, f)$ reactions are presented in section 3. We conclude with recommendations to improve these calculations in the future. The main results of this report can be found in Figs. 3 and 4.

\section{Theory}

\section{$2.1 \quad$ Overview}

The microscopic calculation can be broken down into several sequential steps. We summarize these steps below before discussing them in more detail:

1. Hartree-Fock-Bogoliubov (HFB) calculations, constrained by the new collective coordinates $d$ and $\xi$, are performed to construct a potential energy surface $V(d, \xi)$ as a function of those coordinates

2. The collective inertia tensor is calculated from the HFB solutions for each $(d, \xi)$ configuration

3. Another potential surface $V_{0}(d, \xi)$ is formed to represent the initial potential seen by the fissioning nucleus, by extrapolating $V(d, \xi)$ at the second barrier with a quadratic function of $d$ to form a potential well encompassing the first two barriers.

4. A static collective Schrödinger equation is solved in the $V_{0}(d, \xi)$ well to produce a spectrum of quasi-stationary collective eigenstates.

5. For a given incident neutron energy $E_{n}$ an appropriate initial wave packet is constructed from the quasi stationary states.

6. A time-dependent collective Schrödinger equation, formed using the potential surface and inertia tensor obtained in steps 1 and 2 , is solved numerically to evolve the initial wave packet to scission

7. The wave function flux is integrated over time along a line of $(d, \xi)$ configurations just before scission to produce the mass distribution of the fragments.

\subsection{Choice of collective coordinates}

For a nuclear configuration described in cylindrical coordinates by the density $\rho(r, \varphi, z)$, and with the $z$ axis taken along the symmetry axis of the nucleus, we can define the number of particles to the left and right of a neck position $z_{N}$ by

$$
\begin{aligned}
& A_{L} \equiv \int_{0}^{2 \pi} d \varphi \int_{0}^{\infty} r d r \int_{-\infty}^{z_{N}} d z \rho(r, \varphi, z) \\
& A_{R} \equiv \int_{0}^{2 \pi} d \varphi \int_{0}^{\infty} r d r \int_{z_{N}}^{\infty} d z \rho(r, \varphi, z)
\end{aligned}
$$


with $A_{L}+A_{R}=A_{t o t}$, the total number of nucleons in the fissioning nucleus. We also define the number of particles in the neck by $[5,19]$

$$
Q_{N} \equiv \int_{0}^{2 \pi} d \varphi \int_{0}^{\infty} r d r \int_{-\infty}^{\infty} d z \rho(r, \varphi, z) \exp \left[-\frac{\left(z-z_{N}\right)^{2}}{a_{N}^{2}}\right]
$$

with $a_{N}=1 \mathrm{fm}$. In practice the neck position $z_{N}$ is chosen at the point between the fragments where $Q_{N}$ is a minimum. The collective coordinate $\xi$ is now defined as the difference between the mass numbers of the fragments, normalized by the total number of particles in the fissioning nucleus,

$$
\xi \equiv \frac{A_{R}-A_{L}}{A}
$$

with this definition we always have $-1 \leq \xi \leq 1$ with $\xi<0$ simply giving the mirror image of the corresponding $\xi>0$ solution. The $\xi=0$ case describes symmetric fission.

Next we calculate the center of mass of each fragment

$$
\begin{aligned}
z_{L} & \equiv \frac{1}{A_{L}} \int_{0}^{2 \pi} d \varphi \int_{0}^{\infty} r d r \int_{-\infty}^{z_{N}} d z \rho(r, \varphi, z) z \\
z_{R} & \equiv \frac{1}{A_{R}} \int_{0}^{2 \pi} d \varphi \int_{0}^{\infty} r d r \int_{z_{N}}^{\infty} d z \rho(r, \varphi, z) z
\end{aligned}
$$

and define the collective coordinate $d$ as the distance between those centroids

$$
d \equiv z_{R}-z_{L}
$$

Roughly speaking, the coordinate $d$ replaces $Q_{20}$ and $\xi$ replaces $Q_{30}$ in fission calculations, but these sets of coordinates are not equivalent. As we will see in section 3, the potential surface $V(d, \xi)$ has both similarities with and significant differences from the more traditional $V\left(Q_{20}, Q_{30}\right)$ surface.

\subsection{The collective Hamiltonian}

The collective Hamiltonian is discussed in $[6]$. For the $(d, \xi)$ coordinates used in the present work, the collective Hamiltonian takes the form ${ }^{1}$

$$
H_{\text {coll }}=-\frac{1}{2} \sum_{x=d, \xi} \sum_{y=d, \xi} \frac{\partial}{\partial x} B_{x y}(d, \xi) \frac{\partial}{\partial y}+V(d, \xi)
$$

where the $B_{x y}(d, \xi)$ are elements of the $2 \times 2$ inertia tensor $B(d, \xi)$. The calculation of the $B_{x y}(d, \xi)$ from the HFB solutions, is performed using the same formalism as for the $B_{i j}\left(Q_{20}, Q_{30}\right)$ in [6]. In short, the inertia tensor $B$ is the inverse of the mass tensor $\mathcal{M}$,

$$
B(d, \xi)=\mathcal{M}^{-1}(d, \xi)
$$

\footnotetext{
${ }^{1}$ The TDGCM calculations in the present work do not include zero-point energy corrections.
} 
whose elements are expressed in terms of moments $\mathcal{M}_{x y}^{(-k)}$,

$$
\mathcal{M}_{x y}=\sum_{r, s=d, \xi}\left(\mathcal{M}^{(-1)}\right)_{x r}^{-1}\left(\mathcal{M}^{(-3)}\right)_{r s}\left(\mathcal{M}^{(-1)}\right)_{s y}^{-1}
$$

The moments themselves are calculated from the HFB solutions $|\Phi(d, \xi)\rangle$ and the two quasiparticle states $|\mu \nu\rangle$ built on those HFB states,

$$
\mathcal{M}_{x y}^{(-k)} \equiv \sum_{\mu \nu} \frac{\langle\Phi(d, \xi)|\hat{x}| \mu \nu\rangle\langle\mu \nu|\hat{y}| \Phi(d, \xi)\rangle}{\left(E_{\mu}+E_{\nu}\right)^{k}}
$$

with $E_{\mu}+E_{\nu}$ the energy of the two-quasiparticle state. The operators $\hat{x}$ and $\hat{y}$ stand in for the operators corresponding to the $d$ and $\xi$ coordinates.

As a cross-check of the inertia tensor calculations, we note that for very large separation distance between the fragments, the kinetic energy of the system is governed by the constant reduced mass $\mu$ of the system

$$
-\frac{1}{2} B_{d d} \frac{\partial^{2}}{\partial d^{2}} \longrightarrow \frac{\hbar^{2}}{2 \mu m} p_{d}^{2}
$$

with $m$ the nucleon mass and where the momentum in the $d$ coordinate is given by

$$
p_{d}=\frac{\hbar}{i} \frac{\partial}{\partial d}
$$

From this we deduce the simple asymptotic formula

$$
B_{d d} \longrightarrow \frac{(\hbar c)^{2}}{\mu m c^{2}}
$$

For example, in the case of ${ }^{240} \mathrm{Pu}$ fission, typical mass divisions give $\mu \approx 60$ and therefore $B_{d d} \longrightarrow 0.7 \mathrm{MeV} \mathrm{fm}^{2}$. Our microscopic calculations for the symmetric and $107 / 133$ (i.e., most likely) mass divisions at $d=20 \mathrm{fm}$ also give $B_{d d} \approx$ $0.7 \mathrm{MeV} \mathrm{fm}^{2}$.

\subsection{The initial state}

In order to calculate the quasi-stationary states used to construct the initial wave packet, the potential energy surface $V(d, \xi)$ was modified at the second saddle to create a potential well $V_{0}(d, \xi)$ with high walls. Fig. 1 shows a cut of the modified potential surface for symmetric fission. The same inertia tensor described in section 2.3 is used to calculate the quasi-stationary states by solving the time-independent collective Schrödinger equation

$$
H_{\text {coll }}^{(0)} g_{0}(d, \xi)=E g_{0}(d, \xi)
$$

with

$$
H_{\text {coll }}^{(0)}=-\frac{1}{2} \sum_{x=d, \xi} \sum_{y=d, \xi} \frac{\partial}{\partial x} B_{x y}(d, \xi) \frac{\partial}{\partial y}+V_{0}(d, \xi)
$$




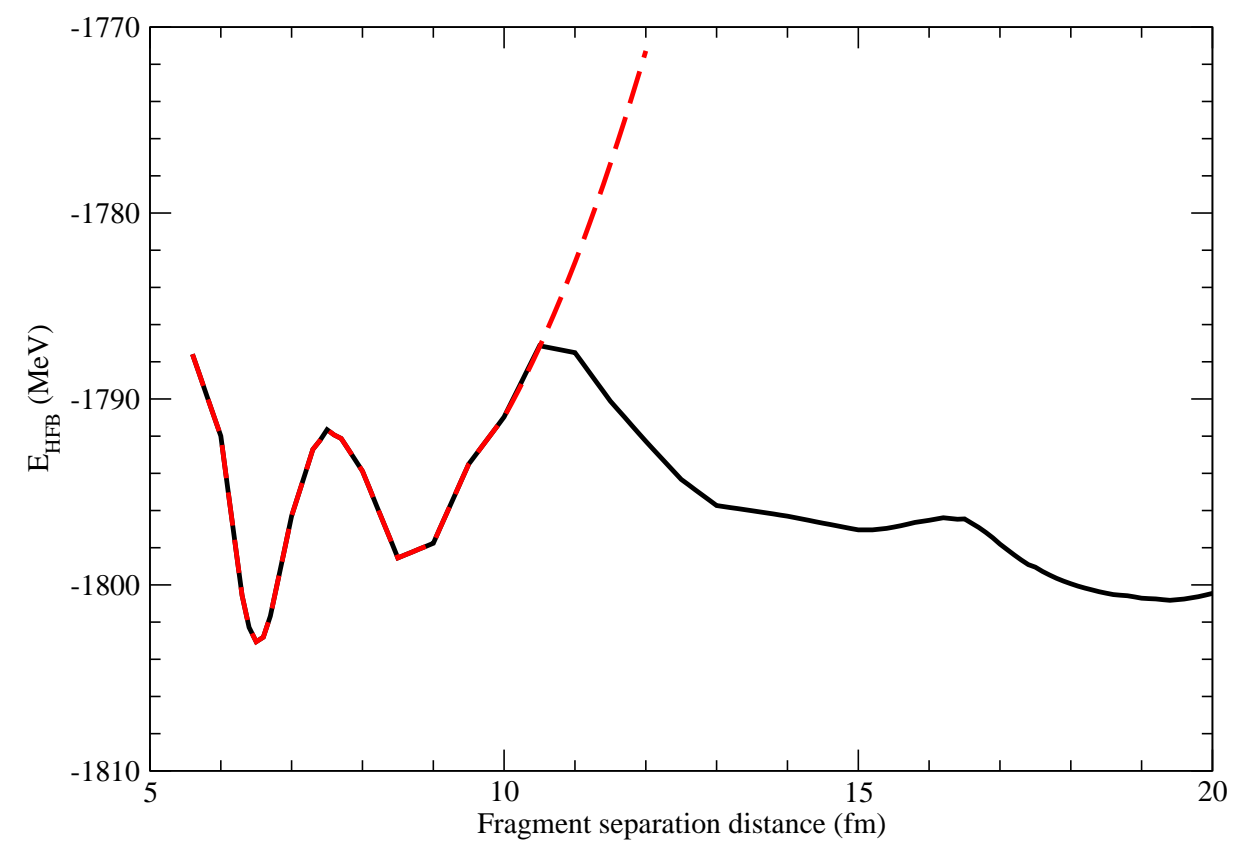

Figure 1: Extended potential (red dashed line) for symmetric fission, used to calculated the quasi-stationary states for ${ }^{240} \mathrm{Pu}$. The black solid line shows the unmodified potential for reference.

the eigenstates $g_{0}(d, \xi)$ and corresponding eigenvalues $E$ are obtained by solving the Schrödinger equation numerically.

For a given incident neutron energy $E_{n}$ we first need to calculate the excitation energy $E_{x}$ of the fissioning system. This excitation energy is given by

$$
E_{x}=E_{n}+E_{A}-S_{n}+\Delta \mathrm{ZPE}
$$

where $E_{A}$ is the height of the first saddle, $S_{n}$ is the separation energy of the neutron in the compound nucleus, and $\triangle \mathrm{ZPE}$ is the difference in zero-point energy (ZPE) between the first well and the first saddle. In practice, we find that the ZPE is essentially the same in the first well and at the first saddle, and therefore $\Delta \mathrm{ZPE} \approx 0$. The barrier heights $E_{A}$ can be found in [20], thus we 
obtain

$$
E_{A}-S_{n}= \begin{cases}0.80 \mathrm{MeV} & \text { for }{ }^{236} \mathrm{U} \\ 0.53 \mathrm{MeV} & \text { for }{ }^{240} \mathrm{Pu}\end{cases}
$$

For each $E_{n}$, a collective wave packet was constructed as the superposition of quasi-stationary states within $500 \mathrm{keV}$ of the corresponding $E_{x}$. Thus, between 8 and 15 equally-weighted states were used for the different energies and the two $(n, f)$ reactions considered here.

\subsection{Calculation of the pre-neutron fragment distributions}

The time dependent collective Schrödinger equation

$$
H_{\text {coll }} g(d, \xi, t)=E g(d, \xi, t)
$$

is solved numerically with the initial condition given by the quasi-stationary states (Eq. (1)),

$$
g(d, \xi, t=0)=g_{0}(d, \xi)
$$

The corresponding probability current $\vec{J}$ is defined at every point $(d, \xi)$ by the continuity equation

$$
\frac{d}{d t}|g(d, \xi, t)|^{2}+\nabla \cdot \vec{J}(d, \xi, t)=0
$$

For each value of $\xi$, we can identify a distance $d_{s}$ such that the nucleus is scissioned for $d>d_{s}$ but not for $d \leq d_{s}$. The resulting "fission line" defined by

$$
d=d_{s}(\xi)
$$

separates the $(d, \xi)$ into inner and outer regions for the time-dependent calculation. The fragment mass distribution before neutron emission is obtained by integrating the flux over time along the fission line

$$
Y(A)=\int_{0}^{\infty} d t \vec{J}(d, \xi, t) \cdot \hat{n} d s
$$

where $\hat{n}$ is a unit normal vector and $d s$ is the length of the infinitesimal segment through which the flux is calculated. In the present work, $\hat{n}$ was taken in the direction of the current $\vec{J}$, which maximizes the flux, and $d s$ was assumed to have the same value for each fragment mass $A$. The yield $Y(A)$ was normalized according to standard convention so that

$$
\sum_{A=0}^{A_{\text {tot }}} Y(A)=200
$$

Finally, we must account for fluctuations in particle number of the fragments due to both pairing effects, and the finite number of particles $Q_{N}$ in the neck 
region for points along the fission line. We find typically $2 \lesssim Q_{N} \lesssim 5$ along the fission line, and have therefore adopted an average value of 3.5 for the fluctuation in the number of particles $A$ in each fragment. This fluctuation leads to a smoothing of the yield which we calculate as

$$
\tilde{Y}(A) \equiv \int_{-\infty}^{\infty} d A^{\prime} Y\left(A^{\prime}\right) \frac{1}{\sqrt{2 \pi} \sigma} \exp \left[-\frac{\left(A-A^{\prime}\right)^{2}}{2 \sigma^{2}}\right]
$$

with $\sigma=3.5$.

\section{Discussion}

The potential energy surface for ${ }^{240} \mathrm{Pu}$ obtained by HFB calculations is plotted as a function of both $\left(Q_{20}, Q_{30}\right)$ and $(d, \xi)$ collective coordinates in Fig. 2. The energy surfaces in both coordinate systems display common features, such as two saddles and two minima in the low $Q_{20}$ or $d$ region, as expected. However, the potential $V(d, \xi)$ is much flatter as a function of $\xi$ starting at the second well and for higher $d$ compared to $V\left(Q_{20}, Q_{30}\right)$ as a function of $Q_{30}$. This "flatness" of the potential is in part responsible for a more slowly falling yield in the wings of the distribution compared to the earlier calculations in [6].

In Figs. 3 and 4 we show the calculated pre-neutron fragment distributions as a function of fragment mass and for different incident neutron energies, obtained for the reactions ${ }^{235} \mathrm{U}(n, f)$ and ${ }^{239} \mathrm{Pu}(n, f)$ respectively. For the ${ }^{235} \mathrm{U}(n, f)$ reaction, pre-neutron yields have been extracted from experimental data by Straede et al. [21], and we compare our calculations directly to those values in Fig. 3. About $2 / 3$ of the calculated fragment yields lie within $30 \%$ of the experimental values, with the most significant discrepancies occurring near peak for thermal fission $\left(E_{n}=0.0 \mathrm{MeV}\right)$ and near symmetric fission $(A=118)$ for $E_{n} \geq 2 \mathrm{MeV}$.

The results in Fig. 4 for the ${ }^{239} \mathrm{Pu}(n, f)$ reaction can only be compared to pre-neutron yields extracted from measurements at thermal neutron energies [22]. In that case, the agreement with experiment is better than for the ${ }^{235} \mathrm{U}(n, f)$ results. About $3 / 4$ of the calculated thermal yields agree with the experimental values to within $30 \%$. There are no experimental data sets available at higher incident neutron energies, and we compare our calculations instead to the phenomenological general fission model (GEF) of Schmidt et al. [16]. As in the ${ }^{235} \mathrm{U}(n, f)$ case, our ${ }^{239} \mathrm{Pu}(n, f)$ yield calculations underestimate the symmetric channel production with increasing $E_{n}$. In addition, the microscopic predictions and GEF results differ in the wings of the distributions for $E_{n} \geq 1 \mathrm{MeV}$.

The results shown in Figs. 3 and 4 represent the best predictions of preneutron mass yields that can be achieved with microscopic theories today. Furthermore, they are comparable (if not better) than some of the phenomenological models that have been fitted to the available data: for example, the GEF model reproduces the experimental Straede et al. data for ${ }^{235} \mathrm{U}(n, f)$ to within $30 \%$ 

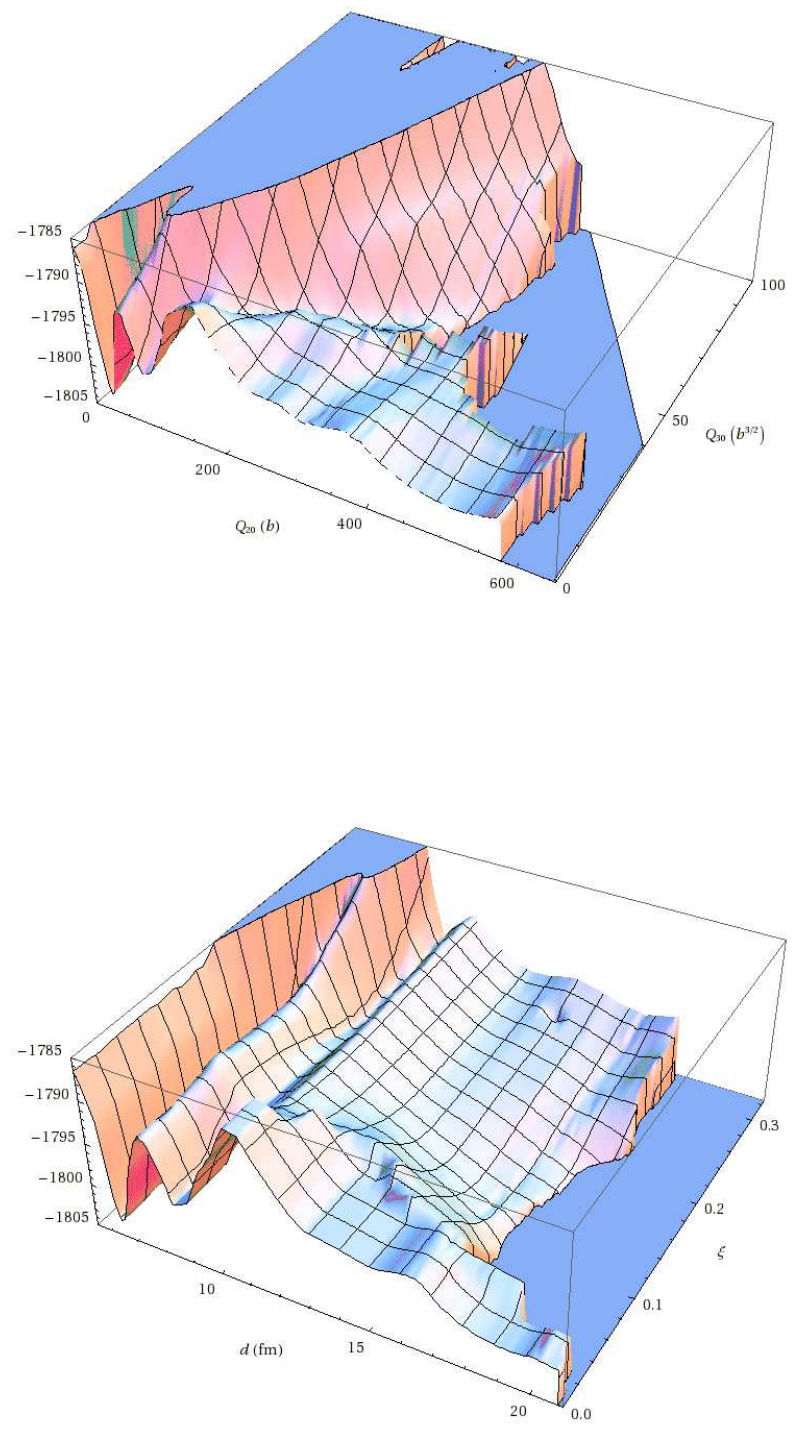

Figure 2: Comparison of ${ }^{240} \mathrm{Pu}$ potential energy surfaces (in $\mathrm{MeV}$ ) calculated as a function of the $\left(Q_{20}, Q_{30}\right)$ coordinates (top plot) and $(d, \xi)$ coordinates (bottom plot). 


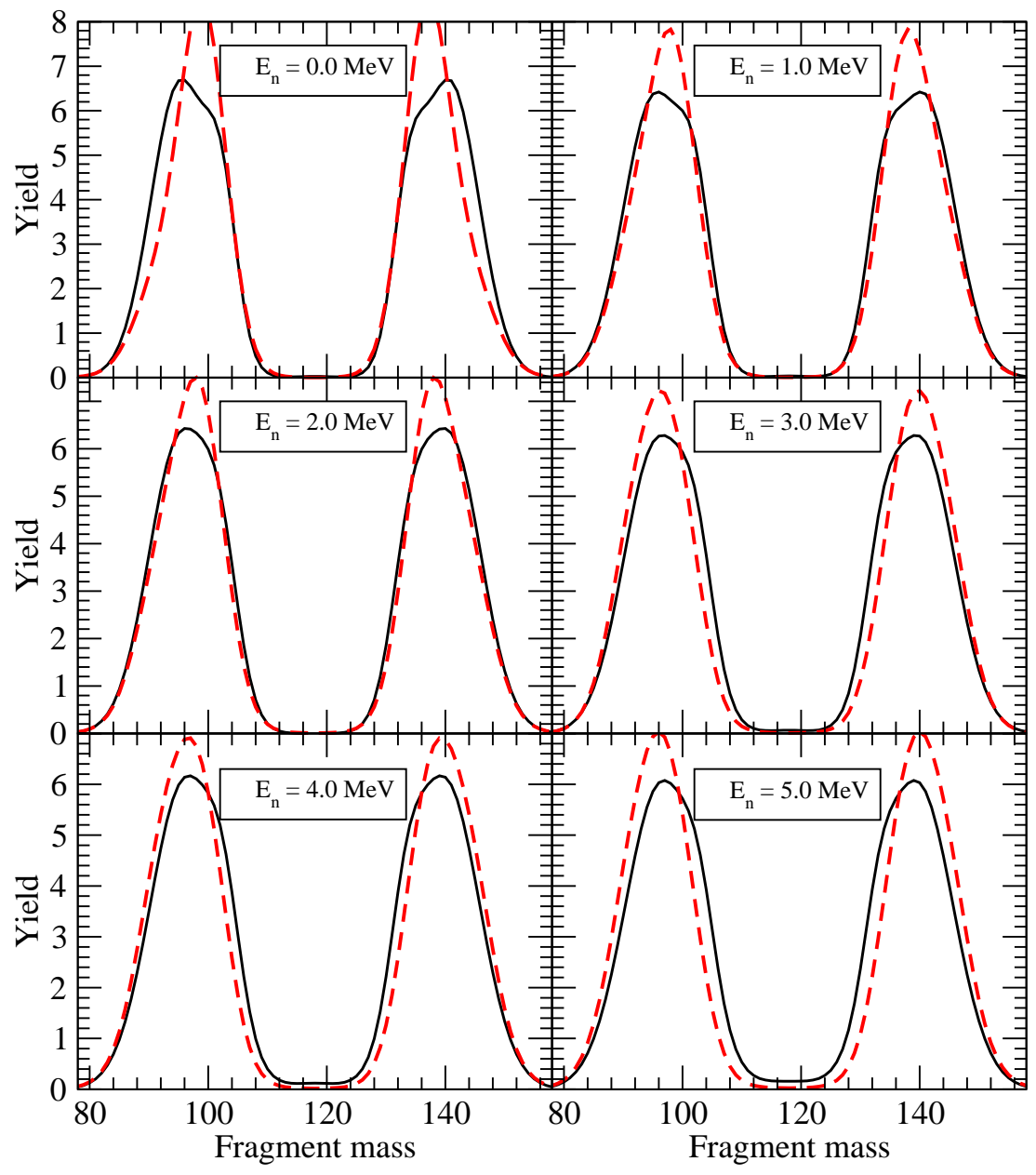

Figure 3: Theoretical calculations of mass yields, before neutron emission, for the ${ }^{235} \mathrm{U}(n, f)$ reaction. Microscopic-theory values (dashed red lines) are compared to the experimental systematics of Straede et al. (solid black curves) at incident neutron energies ranging from $E_{n}=0.0 \mathrm{MeV}$ (thermal), to $E_{n}=5.0$ $\mathrm{MeV}$. 


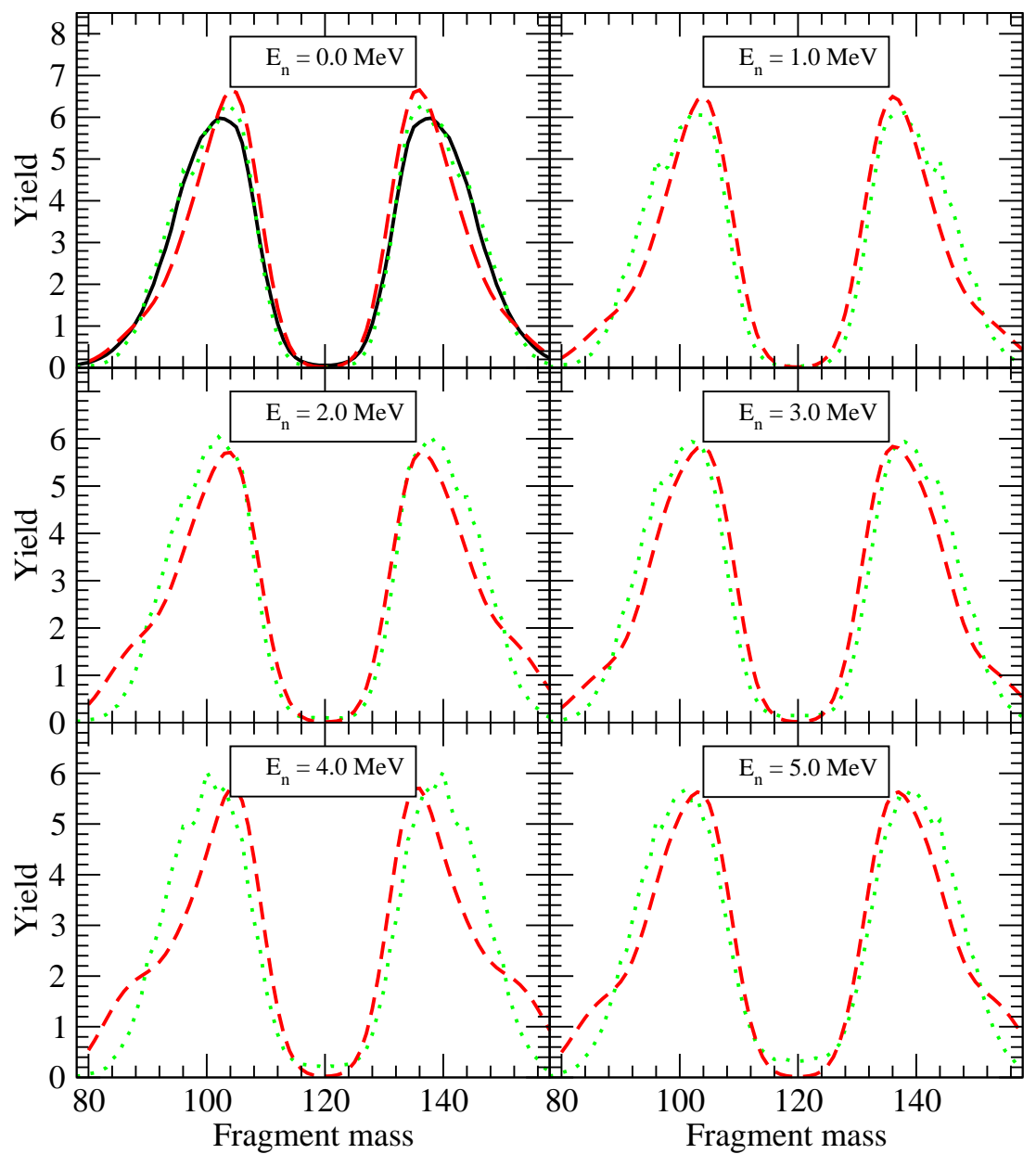

Figure 4: Theoretical calculations of mass yields, before neutron emission, for the ${ }^{239} \mathrm{Pu}(n, f)$ reaction. For thermal neutrons $\left(E_{n}=0.0 \mathrm{MeV}\right)$, microscopictheory values (dashed red lines) are compared to the experimental results of Schillebeeckx et al. (solid black curves) and to calculations using the phenomenological model GEF of Schmidt et al. (dotted green line). For $E_{n}=1.0$ to $5.0 \mathrm{MeV}$, the microscopic-theory calculations are compared to the GEF model results only, since no experimental data are available. 
in about $45 \%$ of the cases shown in Fig. 3, and in about $57 \%$ of the thermalfission yields for ${ }^{239} \mathrm{Pu}(n, f)$. It is worth mentioning that phenomenological approaches in general, because they contain parameters adjusted to the very types of data they are trying to reproduce, should be inherently more flexible than microscopic theories. Nevertheless, the microscopic calculations presented here could be improved to reduce the $30 \%$ agreement with data we are currently able to achieve.

The most important improvement to the calculation concerns the finite-range effective interaction that is the only phenomenological input to the theory. It is well-known [4] that the height of the second saddle depends sensitively on the surface coefficient of the interaction. This parameter is not well-constrained experimentally, and it should be possible to lower the barrier for symmetric fission sufficiently to significantly improve the predicted yields near symmetric fission in Figs. 3 and 4. Furthermore, the asymmetry coefficient of the interaction can also be legitimately adjusted, and could improve the yields for those fragments far from stability. Since the fragments furthest from stability tend to be those near symmetric and very asymmetric fission, this could improve the calculated yield curves near the center and in the wings.

Finally, the TDGCM calculations performed in the present work assume that the potential surface and inertia tensor do not vary with increasing incident neutron energy. The extent to which this assumption is valid is currently being studied at LLNL and elsewhere using collective-intrinsic coupling formalisms [23] and temperature-dependent HFB calculations. These energy-dependent calculations are not expected to drastically alter the results given the relatively low neutron energies considered here $\left(E_{n} \leq 5 \mathrm{MeV}\right)$, but the effects on the yields as a function of $E_{n}$ should nevertheless be explored.

\section{Conclusion}

We have presented microscopic time-dependent calculations of the pre-neutron fragment yields for the ${ }^{235} \mathrm{U}(n, f)$ and ${ }^{239} \mathrm{Pu}(n, f)$ reactions, as a function of incident neutron energies up to $E_{n}=5 \mathrm{MeV}$. Wherever experimental data are available, we find a $\sim 30 \%$ agreement between theory and data for most of the yields we calculate. The present results represent a significant improvement over earlier microscopic calculations which typically differed from experimental values by a factor of two or more. The improvement over those earlier results was achieved primarily by using collective coordinates and corresponding operators more appropriate to the description of the nucleus near scission, than what is commonly used in the literature. Instead of the standard quadrupole and octupole moments of the nucleus, we used the mass difference between fragments and the separation between their centers of mass as collective coordinates.

In the future, the current $30 \%$ discrepancy between theory and data could be further reduced by adjusting the surface and asymmetry coefficients of the effective interaction used in the microscopic calculation. These adjustments could probably increase the calculated yields for symmetric and very asymmetric 
fission and therefore, by normalization of the overall yield distribution, reduce

the yields near peak as well thereby bringing the yield curves in better agreement with experiment.

\section{References}

[1] M. Bender, P.-H. Heenen, and P.-G. Reinhard, Rev. Mod. Phys. 75, 121 (2003).

[2] J.-P. Delaroche, M.Girod, H. Goutte, and J. Libert, Nucl. Phys A771, 103 (2006).

[3] J.-P. Delaroche, M. Girod, J. Libert, H. Goutte, S. Hilaire, S. Péru, N. Pillet, and G. F. Bertsch, Phys. Rev. C 81, 014303 (2010).

[4] J.-F. Berger, M. Girod, and D. Gogny, Nucl. Phys. A428, 23c (1984).

[5] M. Warda, J. L. Egido, L. M. Robledo, and K. Pomorski, Phys. Rev. C 66, 014310 (2002).

[6] H. Goutte, J.-F. Berger, P. Casoli, and D. Gogny, Phys. Rev. C 71, 024316 (2005).

[7] J. C. Pei, W. Nazarewicz, J. A. Sheikh, and A. K. Kerman, Phys. Rev. Lett 102, 192501 (2009).

[8] W. Younes and D. Gogny, Phys. Rev. Lett. 107, 132501 (2011).

[9] A. V. Karpov, P. N. Nadtochy, D. V. Vanin, and G. D. Adeev, Phys. Rev. C 63, 054610 (2001).

[10] P. N. Nadtochy, A. Kelic, K.-H. Schmidt, Phys. Rev. C 75, 064614 (2007).

[11] M. V. Borunov, P. N. Nadtochy, G. D. Adeev, Nucl. Phys. A799, 56 (2008).

[12] J. Randrup and P. Möller, Phys. Rev. Lett. 106, 132503 (2011).

[13] J. Randrup, P. Möller, and A. J. Sierk, Phys. Rev. C 84, 034613 (2011).

[14] J. Decharge and D. Gogny, Phys. Rev. C 21, 1568 (1980).

[15] J.-F. Berger, M. Girod, and D. Gogny, Comp. Phys. Comm. 63, 365 (1991).

[16] K.-H. Schmidt and B. Jurado, JEF/DOC 1423 (2011).

[17] N. Dubray, H. Goutte, and J.-P. Delaroche, Phys. Rev. C 77, 014310 (2008).

[18] W. Younes and D. gogny, "Microscopic Calculation of Fission Fragment Energies for the $239 \mathrm{Pu}($ nth,f) reaction", LLNL-TR-502781 (2011).

[19] W. Younes and D. Gogny, Phys. Rev. C 80, 054313 (2009). 
[20] H. C. Britt, from Preprint LA-4R-79-533 (Review at IAEA Fission Conference, Jülich, 1979).

[21] Ch. Straede, C. Budtz-Jorgensen, and H.-H. Knitter, Nucl. Phys. A462, 85 (1987).

[22] P. Schillebeeckx, C. Wagemans, A. J. Deruytter, and R. Barthélémy, Nucl. Phys. A545, 623 (1992).

[23] R. Bernard, H. Goutte, D. Gogny, and W. Younes, Phys. Rev. C 84, 044308 (2011). 